Lee Peng Yee and Zhao Dongsheng, Division of Mathematics, School of Science, National Institute of Education, Singapore 259756

e-mail: zhaod@am.nie.ac.sg

\title{
UPPER AND LOWER HENSTOCK INTEGRALS
}

\begin{abstract}
The Riemann integral can be defined in terms of upper and lower Riemann sums. In this paper, we show how the Henstock integral can also be defined in a similar manner.
\end{abstract}

A natural generalization of the Riemann integral is the Henstock integral (see, for example, $[\mathbf{2}]$ ), which also includes the Lebesgue integral and the Newton integral. The Henstock integral is also known as the Kurzweil or Kurzweil-Henstock integral. Since the Riemann integral is defined for bounded functions only, upper and lower Riemann sums always exist. However this is no longer the case for the Henstock integral, since Henstock integrable functions are not necessarily bounded. The essential idea in the definition of the Henstock integral is that when forming Riemann sums we do not use all the points in each interval of a partition as it is done in the Riemann integral, rather we use only certain designated points in the interval. This is done by introducing a positive function $\delta(x)$, and using only those points $\xi$ in $[u, v]$ for which $[u, v] \subset(\xi-\delta(\xi), \xi+\delta(\xi))$. Keeping to such $\xi$, a kind of upper and lower sums can now be define. In this paper, we identify the designated points directly by introducing a contraction of intervals into their subsets, which serves the same purpose as the positive function $\delta(\xi)$ in the Henstock integral, and thus define corresponding upper and lower sums and in turn the upper and lower Henstock integrals. When the two integrals are equal, we obtain the Henstock integral.

We recall that given $\delta(\xi)>0$, a partition $D$ of $[0,1]$ given by

$$
x_{0}=0<x_{1}<\cdots<x_{n}=1, \xi_{1}, \xi_{2}, \cdots, \xi_{n},
$$

Key Words: Henstock integrals, Riemann sums, contraction

Mathematical Reviews subject classification: 25A39

Received by the editors September 16, 1996 
is said to be $\delta$-fine if $\xi_{i} \in\left[x_{i-1}, x_{i}\right] \subset\left(\xi_{i}-\delta\left(\xi_{i}\right), \xi_{i}+\delta\left(\xi_{i}\right)\right)$ for $i=1,2, \cdots, n$. Then a function $f$ is Henstock integrable to $A$ on $[0,1]$ if for every $\epsilon>0$ there is $\delta(\xi)>0$ such that for every $\delta$-fine partition $D$ of $[0,1]$ we have

$$
\left|\sum_{i=1}^{n} f\left(\xi_{i}\right)\left(x_{i}-x_{i-1}\right)-A\right|<\epsilon .
$$

For convenience, we write $D=\{([u, v], \xi)\}$ where $[u, v]$ denotes a typical interval in $D$ and $\xi \in[u, v]$. Then for brevity we write the above inequality as

$$
\left|(D) \sum f(\xi)(v-u)-A\right|<\epsilon
$$

A set-valued function $\nu$ defined on the set of all nondegenerate closed subintervals of $[0,1]$ is called a contraction on $[0,1]$ if it maps a nondegenerate closed interval $I \subseteq[0,1]$ to a subset $\nu(I)$ of $[0,1]$, possibly empty, such that the following conditions are satisfied:

(1) $\nu(I) \subseteq I$ for each interval $I \subseteq[0,1]$,

(2) $I_{1} \subseteq I_{2}$ implies $\nu\left(I_{2}\right) \cap I_{1} \subseteq \nu\left(I_{1}\right)$, (3) $\bigcup_{I \subseteq[0,1]}\left(\nu(I) \cap I^{o}\right)=[0,1]$, where $I^{o}$ denotes the interior of $I$, except when

$I=[0, a]$ or $[b, 1]$, then $I^{o}$ means $[0, a)$ or $(b, 1]$ respectively.

A trivial example is when $\nu(I)=I$. However, very often, $\nu(I)$ is a proper subset of $I$.

Lemma 1. Let $\nu_{1}$ and $\nu_{2}$ be two contractions on $[0,1]$. Then $\nu(I)=\nu_{1}(I) \cap$ $\nu_{2}(I)$, for all intervals $I \subseteq[0,1]$, is also a contraction on $[0,1]$.

Proof. Conditions (1) and (2) in the definition are clearly true for $\nu$. Now we prove only (3). Consider any two intervals $I$ and $J$ inside $[0,1]$. Since $\nu_{1}(I) \subseteq I$ and $\nu_{2}(J) \subseteq J$, we have $\nu_{1}(I) \cap \nu_{2}(J)=\left(\nu_{1}(I) \cap I \cap J\right) \cap\left(\nu_{2}(J) \cap I \cap J\right) \subseteq$ $\nu_{1}(I \cap J) \cap \nu_{2}(I \cap J)=\nu(I \cap J)$. This implies that

$$
\left(\nu_{1}(I) \cap I^{o}\right) \cap\left(\nu_{2}(J) \cap J^{o}\right) \subseteq \nu(I \cap J) \cap(I \cap J)^{o} .
$$

Then

$$
[0,1]=\left(\bigcup_{I \subseteq[0,1]} \nu_{1}(I) \cap I^{o}\right) \bigcap\left(\bigcup_{J \subseteq[0,1]} \nu_{2}(J) \cap J^{o}\right)
$$




$$
\begin{aligned}
& =\bigcup_{I, J \subseteq[0,1]}\left(\nu_{1}(I) \cap \nu_{2}(J) \cap I^{o} \cap J^{o}\right) \\
& \subseteq \bigcup_{I, J \subseteq[0,1]}\left(\nu(I \cap J) \cap(I \cap J)^{o}\right) \\
& =\bigcup_{I \subseteq[0,1]}\left(\nu(I) \cap I^{o}\right) .
\end{aligned}
$$

For any two contractions $\nu_{1}$ and $\nu_{2}$, we write $\nu_{1} \leq \nu_{2}$ if $\nu_{2}(I) \subseteq \nu_{1}(I)$ for all intervals $I \subseteq[0,1]$. In view of the above lemma, the family of all contractions is a directed set with respect to the order $\leq$ and hence we may define the Moore-Smith limit using this directed set. For Moore-Smith or generalized limit, see [1].

A partition $D$ of $[0,1]$ is said to be $\nu$-fine if $\nu([u, v])$ is nonempty for every $[u, v]$ in $D$. Let $\nu$ be a contraction on $[0,1]$. We define the following sums if they exist.

$$
S^{l}(D, f)=(D) \sum \inf _{\eta \in \nu([u, v])} f(\eta)(v-u), \quad S^{l}(\nu, f)=\inf _{D} S^{l}(D, f) .
$$

where $S^{l}(D, f)$ corresponds to the lower Riemann sum of $f$ except that the infimum is now taken over $\nu([u, v])$ and not the whole interval $[u, v]$, and the infimum in $S^{l}(\nu, f)$ is taken over all $\nu$-fine partitions $D$ of $[0,1]$. Note that $S^{l}(D, f)$ has a meaning only when $f$ is bounded on $\nu([u, v])$. Similarly, we define

$$
S^{u}(D, f)=(D) \sum \sup _{\eta \in \nu([u, v])} f(\eta)(v-u), \quad S^{u}(\nu, f)=\sup _{D} S^{u}(D, f) .
$$

In fact, $S^{l}(\nu, f)$ and $S^{u}(\nu, f)$ can also be obtained in one step as follows:

$$
S^{l}(\nu, f)=\inf _{D}(D) \sum f(\xi)(v-u) \text { and } S^{u}(\nu, f)=\sup _{D}(D) \sum f(\xi)(v-u),
$$

where the infimum and supremum are taken over all $\nu$-fine partitions $D$ of $[0,1]$ with $\xi \in \nu([u, v])$ for all $[u, v]$ in $D$.

Theorem 2. If a function $f$ is Henstock integrable to $A$ on $[0,1]$, then there is a contraction $\nu$ on $[0,1]$ such that $f$ is bounded on $\nu(I)$ for each $I \subseteq[0,1]$, and furthermore $S^{l}(\nu, f)$ and $S^{u}(\nu, f)$ exist.

Proof. Let $\epsilon=1$ and $\delta(\xi)>0$ be given as in the definition of the Henstock integral, and $F$ the Henstock primitive (or Henstock indefinite integral) of $f$ on 
$[0,1]$. For any interval $I \subseteq[0,1]$, define $\nu_{\delta}(I)=\{x \in I \mid I \subseteq(x-\delta(x), x+\delta(x))\}$. Then $\nu_{\delta}$ is a contraction on $[0,1]$. It follows from Henstock's lemma [2] that if $\nu_{\delta}([u, v]) \neq \emptyset$, then $|f(\xi)(v-u)-F(v)+F(u)| \leq 1$ for $\xi \in \nu_{\delta}([u, v])$. Consequently,

$$
|f(\xi)| \leq \frac{1+|F(v)-F(u)|}{|v-u|} \text { for } \xi \in \nu_{\delta}([u, v]) .
$$

That is, $f$ is bounded on $\nu_{\delta}([u, v])$ when $\nu_{\delta}([u, v]) \neq \emptyset$. The case when $\nu_{\delta}([u, v])=\emptyset$ is trivial. Also, for any $\nu$-fine partition $D$ of $[0,1]$ and any $\xi \in[u, v]$ we have $\left|(D) \sum f(\xi)(v-u)\right| \leq|F(1)-F(0)|+1$. In other words, $S^{l}(\nu, f)$ and $S^{u}(\nu, f)$ exist.

The above theorem provides the necessary motivation. The upper and lower Henstock integrals can now be defined by

$$
(H) \underline{\int f}=\sup _{\nu \geq \nu_{0}} S^{l}(\nu, f) \text { and }(H) \bar{\int} f=\inf _{\nu \geq \nu_{0}} S^{u}(\nu, f),
$$

where $\nu_{0}$ is any contraction on $[0,1]$ so that $S^{l}\left(\nu_{0}, f\right)$ or $S^{u}\left(\nu_{0}, f\right)$ exists in each respective case.

Note that the definitions above are independent of the choice of $\nu_{0}$. In fact, $(H) \underline{\int f}$ is the Moore-Smith limit of $S^{l}(\nu, f)$ with $\nu$ running through the directed set of contractions that are greater than some $\nu_{0}$ for which $S^{l}\left(\nu_{0}, f\right)$ exists. Similarly, $(H) \bar{\int} f$ is the Moore-Smith limit of $S^{u}(\nu, f)$ over $\nu$.

Now we prove the main theorem of this paper.

Theorem 3. The following conditions are equivalent for a function $f$ on $[0,1]$.

(1) $f$ is Henstock integrable to $A$ on $[0,1]$.

(2) For any $\epsilon>0$ there is a contraction $\nu$ on $[0,1]$ such that for any $\nu$ fine partition $D$ of $[0,1]$, we have $\left|(D) \sum f(\xi)(v-u)-A\right|<\epsilon$, where $\xi \in \nu([u, v])$ for all $[u, v] \in D$.

(3) For any $\epsilon>0$ there is a contraction $\nu$ on $[0,1]$, such that for any $\nu$-fine partitions $D_{1}$ and $D_{2}$ of $[0,1]$, we have

$$
\left|\left(D_{1}\right) \sum f(\xi)(v-u)-\left(D_{2}\right) \sum f(\xi)(v-u)\right|<\epsilon .
$$

(4) The upper and lower Henstock integrals exist and are both equal to A. 
(5) Let

$$
\sigma(\nu)=\sup _{D}\left|(D) \sum f(\xi)(v-u)-A\right|,
$$

where the supremum is taken over all $\nu$-fine partitions $D$ of $[0,1]$. Then the Moore-Smith limit of $\sigma(\nu)$ over the directed set of all contractions on $[0,1]$ is 0 .

Proof. Suppose (1) holds. Let $\epsilon$ and $\delta(\xi)>0$ be given as in the definition of the Henstock integral. Define $\nu_{\delta}$ as in the proof of Theorem 2. Then it follows from the definition of the Henstock integral that for any $\nu_{\delta}$-fine partition $D$ of $[0,1]$ we have $\left|(D) \sum f(\xi)(v-u)-A\right|<\epsilon$. Hence (1) implies (2).

The fact that (2) implies (3) is straightforward. Next, suppose (3) holds. Then for any $\nu$-fine partitions $D_{1}$ and $D_{2}$ of $[0,1]$, we have

$$
\left|S^{u}\left(D_{1}, f\right)-S^{l}\left(D_{2}, f\right)\right| \leq \epsilon .
$$

Taking all possible $\nu$-fine partitions $D_{1}$ and $D_{2}$ of $[0,1]$, we have

$$
0 \leq S^{u}(\nu, f)-S^{l}(\nu, f) \leq \epsilon
$$

Note that if $\nu_{1} \leq \nu_{2}$, then $S^{l}\left(\nu_{1}, f\right) \leq S^{l}\left(\nu_{2}, f\right) \leq S^{u}\left(\nu_{2}, f\right) \leq S^{u}\left(\nu_{1}, f\right)$. Finally, we obtain

$$
0 \leq \inf _{\nu \geq \nu_{0}} S^{u}(\nu, f)-\sup _{\nu \geq \nu_{0}} S^{l}(\nu, f) \leq S^{u}(\nu, f)-S^{l}(\nu, f) \leq \epsilon .
$$

That is, (3) implies (4).

Now suppose (4) holds. Then for any contraction $\nu$ on $[0,1]$

$$
\sigma(\nu)=\max \left\{\left|S^{u}(\nu, f)-A\right|,\left|S^{l}(\nu, f)-A\right|\right\} .
$$

Using the fact that the upper and lower Henstock integrals are the MooreSmith limits of $S^{u}(\nu, f)$ and $S^{l}(\nu, f)$ respectively, we obtain that (4) implies (5).

Finally, suppose (5) holds. Then for every $\epsilon>0$ there is a contraction $\nu$ on $[0,1]$ such that $\sigma(\nu)<\epsilon$. For any $\xi \in[0,1]$, in view of condition (3) in the definition of $\nu$ we can find a closed interval $I$ such that $\xi \in \nu(I) \cap I^{o}$. Then we define $\delta(\xi)>0$ such that $(\xi-\delta(\xi), \xi+\delta(\xi)) \subseteq I$. Then any $\delta$-fine partition $D$ of $[0,1]$ is also $\nu$-fine, and we have

$$
\left|(D) \sum f(\xi)(v-u)-A\right| \leq \sigma(\nu)<\epsilon .
$$

As we can see in the proof of Theorem 3, the correspondence between $\delta(\xi)$ and $\nu$ need not be one-to-one. Also, we see that each of (2) to (5) in Theorem 
3 provides an alternative definition to the Henstock integral. In particular, condition (2) and the integral value is uniquely determined in view of Lemma 1. We remark that if we relax the conditions of a contraction to the following:

$\left(1^{\prime}\right) I_{1} \subseteq I_{2}$ implies $\nu\left(I_{2}\right) \subseteq \nu\left(I_{1}\right)$, and

$$
\left(2^{\prime}\right) \bigcup_{I \subseteq[0,1]}\left(\nu(I) \cap I^{o}\right)=[0,1]
$$

then each of the corresponding results in Theorem 3 is equivalent to the McShane integral [2, p. 108].

Next, we give a condition on $\nu$ such that the Henstock integral reduces to the Riemann integral. A contraction $\nu$ on $[0,1]$ is said to be locally open if for any point $x \in[0,1]$, there is a closed interval $I$, such that $x \in(\nu(I))^{\circ}$.

Theorem 4. A function $f$ is Riemann integrable on $[0,1]$ if and only if, for each $\epsilon>0$, there is a locally open contraction $\nu$ on $[0,1]$ such that $f$ is bounded on $\nu(I)$ for each closed interval $I$, and $\left|S^{u}(\nu, f)-S^{l}(\nu, f)\right|<\epsilon$.

Proof If $f$ is Riemann integrable on $[0,1]$, then for any $\epsilon>0$ there is a constant $\delta>0$, such that for any $\delta$-fine partition $D$ of $[0,1]$,

$$
\left|(D) \sum \sup _{x \in[u, v]} f(x)(v-u)-(D) \sum \inf _{y \in[u, v]} f(y)(v-u)\right|<\epsilon .
$$

Let $\nu_{\delta}$ be the contraction on $[0,1]$ generated by $\delta(\xi)$ as in the proof of Theorem 2. Then by the construction of $\nu$ we see that $\nu_{\delta}$ is locally open, and $\left|S^{u}\left(\nu_{\delta}, f\right)-S^{l}\left(\nu_{\delta}, f\right)\right| \leq \epsilon$.

Conversely, suppose $f$ satisfies the condition. Then, for any $\epsilon>0$, we can show that for each point $x$ there is an interval $I_{x}$ with $x \in I_{x}^{o}$ and $\nu\left(I_{x}\right)=I_{x}$. Now $\left\{I_{x}^{o} \mid x \in[0,1]\right\}$ is an open cover of $[0,1]$. So there is a partition $D=$ $\{([u, v], x)\}$ of $[0,1]$ such that each $[u, v]$ is contained in $I_{x}$. Hence $\nu([u, v])=$ $[u, v]$, and so $D$ is a $\nu$-fine partition. Since $f$ is bounded on each interval $[u, v]$, it is a bounded function on $[0,1]$. From the assumption on $\nu$, it follows that $\left|S^{u}(D, f)-S^{l}(D, f)\right|<\epsilon$. Actually, the sums in the above inequality are respectively the upper and lower Riemann sums of $f$ with respect to the partition $D$. Hence $f$ is Riemann integrable on $[0,1]$.

\section{References}

[1] N. Dunford and J. T. Schwartz, Linear Operators I, Interscience 1958.

[2] P. Y. Lee, Lanzhou Lectures in Henstock Integration, World Scientific 1989. 